Viviane dos Santos Augusto ${ }^{1}$, Graziela Saraiva Reis ${ }^{1}$, Maria Eliza Jordani de Souza ${ }^{2}$, Alfredo José Rodrigues $^{3}$, Paulo Roberto Barbosa Evora $^{4}$

1. Pós-graduanda do Departamento de Cirurgia e Anatomia da Faculdade de Medicina de Ribeirão Preto da Universidade de São Paulo - USP Ribeirão Preto (SP), Brasil.

2. Bioquímica do Laboratório de Bioquímica da Divisão de Cirurgia Experimental da Faculdade de Medicina de Ribeirão Preto da Universidade de São Paulo - USP - Ribeirão Preto (SP), Brasil.

3. Doutor, Professor do Departamento de Cirurgia e Anatomia da Faculdade de Medicina de Ribeirão Preto da Universidade de São Paulo - USP Ribeirão Preto (SP), Brasil. 4. Professor Titular, Departamento de Cirurgia e Anatomia da Faculdade de Medicina de Ribeirão Preto da Universidade de São Paulo - USP Ribeirão Preto (SP), Brasil.

Trabalho realizado no Laboratório de Função Endotelial do Departamento de Cirurgia e Anatomia da Faculdade de Medicina de Ribeirão Preto da Universidade de São Paulo - USP Ribeirão Preto (SP), Brasil.

Submetido em 25 de Março de 2008 Aceito em 15 de Abril de 2009

Autor para correspondência: Paulo Roberto Barbosa Evora Rua Rui Barbosa, 367- Ap. 15 CEP: 14015-120 - Ribeirão Preto (SP), Brasil.

Fone/Fax (016) 3610-9890 -

E-mail: prbevora@netsite.com.br

\section{A utilizaçáo do condensado do exalado pulmonar poderá ser incorporada à rotina de unidades de tratamento intensivo?}

\author{
Will use of exhaled breath condensate be useful for the intensive \\ care unit routine?
}

\section{RESUMO}

A produção endógena de óxido nítrico pode ser detectada e monitorada no ar exalado de homens e animais. O óxido nítrico exalado tem se tornado um instrumento valioso de diagnóstico e monitorização da inflamação e estresse oxidativo dos pulmóes. Dessa forma, a presente revisão foi elaborada com a intençáo de discutir o papel do óxido nítrico no condensado do exalado pulmonar como uma ferramenta útil em investigaçóes de disfunçôes respiratórias na unidade de tratamento intensivo. Observa-se, na literatura especializada, um aumento do interesse no uso do condensado do exalado pulmonar como um método não invasivo para investigar doenças pulmonares. Entretanto, praticamente não existe um método padronizado para a sua coleta, ressaltando-se que o uso de vários ta a comparaçáo de diferentes estudos. $\mathrm{O}$ conteúdo da revisão aponta para prováveis dificuldades da utilização rotineira do condensado exalado pulmonar em pacientes internados em unidades de terapia intensiva, principalmente quando submetidos à ventilação mecânica. Estudos específicos e tecnologias ainda são necessários para que o condensado exalado pulmonar se torne uma ferramenta rotineira de pesquisas e monitorizaçáo de pacientes gravemente enfermos com comprometimento do sistema respiratório. A literatura aponta para a realização de esforços nessa direção.

Descritores: Óxido nítrico/uso diagnóstico; Doenças respiratórias/diagnostico; Unidades de terapia intensiva métodos de assistência respiratória dificul-

\section{INTRODUÇÃO}

Furchgott e Zawadski, em 1980 mostraram que os efeitos vasodilatadores da acetilcolina eram dependentes do endotélio vascular e sugeriram a existência de um fator vasodilatador dependente do endotélio, entáo chamado endothelium-derived relaxing factor (EDRF). ${ }^{(1)}$ Esses autores demonstraram que o EDRF era uma molécula lábil e altamente difusível, com meia vida curta de apenas alguns segundos, atuando no relaxamento do músculo liso dos vasos. Comprovou-se, em 1987, que o EDRF era realmente o óxido nítrico (NO).(2)

Há cerca de vinte anos, a pequena e simples molécula de $\mathrm{NO}$ era conhecida somente como um poluente nocivo ao meio ambiente, presente na fumaça do cigarro, em exaustores de automóveis e em combustíveis fósseis, destruindo a camada de ozônio e provocando chuva ácida. ${ }^{(3)}$ Com as descobertas atuais passou a ser visto como uma molécula endógena essencial na fisiologia do corpo humano, inclusive na regulação do sistema respiratório. ${ }^{(4)}$

O NO é importante para o controle fisiológico da função pulmonar e na fisiopatologia de várias doenças pulmonares como: asma, doença pulmonar obstrutiva crônica (DPOC), fibrose cística (FC), síndrome da angústia respiratória aguda (SARA), 
bronquiectasia, doenças intersticiais pulmonares, hipertensão pulmonar (HP), e outras situaçóes que envolvam inflamação crônica e estresse oxidativo. ${ }^{(3,5)}$ A produção endógena de NO pode ser detectada e monitorizada no ar exalado de animais e homens. ${ }^{(6)}$ Nos últimos anos, o NO exalado tem se tornado um instrumento valioso de diagnóstico e monitorização de inflamação das vias aéreas na asma e, pode se tornar importante para avaliação de outras condiçôes inflamatórias, pela análise da composição do epitélio de revestimento do baixo trato respiratório. ${ }^{(5,6)}$

O presente artigo pretende discutir o papel do NO, medido no condensado exalado pulmonar, como uma possível ferramenta para a monitorização do paciente gravemente enfermo, auxiliando no entendimento de importantes processos patológicos. Serão ressaltadas as dificuldades para sua coleta e análise, incluindo a apresentação de experiência pessoal.

\section{Produçáo e funçóes do óxido nítrico}

$\mathrm{O} \mathrm{NO}$ endógeno é derivado do processo de oxidação e desaminação da L-arginina em citrulina e NO, através de um sistema de enzimas chamadas óxido nítrico sintases (NOS). ${ }^{(7-9)}$ Três isoformas de NOS são descritas. ${ }^{(3,4,9)}$ Duas delas são constitutivas: a neuronal (NOS1 ou nNOS) e a endotelial (NOS3 ou eNOS), que se encontram ancoradas na membrana plasmática e produzem $\mathrm{NO}$ em pequenas quantidades, em ritmo fisiológico, e são dependentes de cálcio $\left(\mathrm{Ca}^{++}\right)$- calmodulina. A terceira forma é a induzida (NOS2 ou iNOS) que, independente da concentraçáo de $\mathrm{Ca}^{++}$intracelular, é expressa no organismo mediante apropriada indução (por citocinas inflamatórias, endotoxinas e toxinas bacterianas) e é responsável pela produção de grandes quantidades de NO..$^{(8,9)}$

Dependendo do local de síntese, da quantidade produzida, do tipo de isoenzima envolvida e do microambiente onde vai atuar, o $\mathrm{NO}$ exercerá diferentes açōes. ${ }^{(9-11)} \mathrm{O} \mathrm{NO}$ medeia vários fenômenos, como vasodilatação dependente do endotélio, citotoxicidade mediada por macrófagos, inibição da ativação, adesão e agregação plaquetária. Além disso, atua como neurotransmissor no sistema nervoso central (SNC) e no sistema nervoso periférico (SNP), participando inclusive do aprendizado e da memória. ${ }^{(11,12)}$ No sistema respiratório funciona como broncodilatador ao agir como neurotransmissor da parcela broncodilatadora do sistema não-adrenérgico não-colinérgico (NANC) em oposição aos estímulos colinérgicos broncoconstritores. Por outro lado, o NO produzido pelo epitélio brônquico possui ação relaxadora da musculatura brônquica. Ao ser produzido também pelo endotélio vascular pulmonar, o NO tende a provocar vasodilatação na mucosa brônquica. ${ }^{(11)}$

Três aspectos físicos-químicos do $\mathrm{NO}$ determinam seu papel crucial no trato respiratório e circulação pulmonar, o que tem levado ao desenvolvimento de novas linhas diagnósticas e terapêuticas: a) a reação cinética do $\mathrm{NO}$ e oxigênio na fase gasosa é lenta, produzindo baixa concentração de NO; b) possui alta capacidade de difusão e, c) tem rápida reaçáo com a hemoglobina. Todos esses fatores levam à rápida remoção do $\mathrm{NO}$ dos pulmóes sem efeito sistêmico. Então, a manutenção e compreensão do $\mathrm{NO}$ nas vias aéreas e no tecido pulmonar é ditada, principalmente, pela ligação do NO com a hemoglobina intravascular. ${ }^{(13)}$

Concentraçóes altas de NO na árvore traqueobrônquica induzem a hiperemia, exsudação plasmática, secreção de muco e proliferação linfocitária $\left(\mathrm{TH}_{2}\right)$, os responsáveis pela proliferação eosinofílica, em uma seqüência de acontecimentos que caracteriza os fenômenos inflamatórios. ${ }^{(12,13)}$

\section{Métodos de mensuraçáo do óxido nítrico exalado}

Até meados de 1980 eram usadas técnicas invasivas, como broncoscopia e lavado broncoalveolar, para a coleta e análise do NO exalado. No entanto, eram técnicas desconfortáveis pelo seu poder invasivo, em que os pacientes precisavam ser sedados e envolviam riscos como: broncoconstrição, infecções e reações inflamatórias. ${ }^{(5,14)}$ Assim não podiam ser repetidas em menos de vinte e quatro horas. ${ }^{(14)}$ Foi a partir de 1980, com Kharitonov e Barnes, que surgiram os primeiros estudos sobre uma técnica náo-invasiva para se analisar os marcadores inflamatórios pulmonares, diretamente do ar exalado - condensado do exalado pulmonar (CEP), em que o ar exalado é coletado através da respiraçáo saturada com vapor d'água via sistema refrigerado. ${ }^{(14,15)}$

Embora essa técnica ainda não esteja bem padronizada, nos últimos anos, tem havido um grande interesse pelo meio científico, por ser um método seguro, simples e de baixo custo, podendo ser repetido várias vezes conforme a necessidade. ${ }^{(5)}$ Pode ser usada em neonatos, adultos e idosos, estejam eles conscientes ou submetidos à ventilação mecânica. Além disso, não requer treino específico exaustivo e pode ser realizada com equipamentos portáteis..$^{(16,17)}$

Para mensuraçáo do NO no ar exalado existem duas modalidades: "online" e "offline". O termo online se refere à leitura de NO realizada pelo analisador que ocorre exatamente no momento da coleta, com armazenamento dos dados na memória do próprio aparelho. Já na modalidade offline, o nitrito/nitrato exalado é coletado através de um reservatório com sistema de condensação, permitindo seu armazenamento para posterior análise. ${ }^{(18)}$

Dentre os métodos de análise do NO exalado, o método da quimioluminescência vem sendo bastante utilizado, devido à grande disponibilidade no mercado de aparelhos que utilizam essa técnica para dosagem do $\mathrm{NO}\left(\mathrm{NIOX}^{\circ} \mathrm{NO}\right.$ analyser 
- Aerocrine, Sweden, LR2000 analyser - Logan Research Ltd, Rochester, UK, ECO Physics NO analyser - ECO - PHYSICS, Duernten, Switzerland e Sievers NO analyser - Ionics Instrument, Boulder, USA). ${ }^{(19)}$

\section{Doenças inflamatórias das vias aéreas inferiores e condensado do exalado pulmonar}

$\mathrm{Um}$ as doenças respiratórias incluindo asma, DPOC, FC e bronquiectasias, envolvem inflamação crônica e estresse oxidativo. Muitos mediadores inflamatórios como peróxido de hidrogênio $\left(\mathrm{H}_{2} \mathrm{O}_{2}\right)$, nitrotirosina, leucotrienos, e o próprio $\mathrm{NO}$ sob a forma de seus metabólitos estáveis (NOx - nitrito $\left(\mathrm{NO}_{2}^{-}\right) /$nitrato $\left(\mathrm{NO}_{3}^{-}\right)$, podem ser identificados no CEP dos pacientes portadores dessas doenças..$^{(5,12,15,16)}$ No entanto, o NO tem sido o biomarcador mais estudado, pois ele atua na regulação do tônus da musculatura lisa dos vasos sangüíneos pulmonares e em doenças inflamatórias das vias aéreas, não somente como marcador, mas também apresenta efeitos anti-inflamatórios. ${ }^{(17)}$ Para tanto, critérios metodológicos bem estritos necessitaram ser definidos. Com essa perspectiva, a American Thoracic Society ${ }^{(20)}$ e a European Respiratory Society ${ }^{(21)}$ prepararam sugestôes para o uso adequado do NO como instrumento diagnóstico e de acompanhamento de pacientes. Um dado muito importante merece destaque: ainda não se tem certeza de que o valor medido corresponde ao que realmente está sendo produzido na via aérea. ${ }^{(22,23)}$

No caso de se utilizar a medida do NO do CEP para monitorização, experimental ou clínica, anestesiologistas e intensivistas terão, obrigatoriamente que vivenciar as possíveis interferências, das acima mencionadas doenças respiratórias, nas concentrações do NO do CEP.

\section{Hipertensão pulmonar, síndrome da angústia respi- ratória aguda e pneumonia}

Dois assuntos são de particular interesse em terapia intensiva: a hipertensão pulmonar (HP) e a síndrome da angústia respiratória aguda (SARA).

A patogênese da HP permanece pobremente estudada. A vasoconstrição é provavelmente o maior fator nos estágios iniciais da doença, e uma reduçáo do $\mathrm{NO}$ endógeno pode contribuir para o seu desenvolvimento. ${ }^{(5,11)}$ De fato, a nebulizaçáo com epoprostenol aumenta o $\mathrm{NO}$ exalado em pacientes com HP, mas não aumenta em controles normais, sugerindo que este efeito na circulação hipertensiva tem um mecanismo relacionado com o NO. Em contraste, a inibiçáo da enzima conversora de angiotensina (ECA) pelo enalapril, usado no tratamento de $\mathrm{HP}$, aumenta os níveis de $\mathrm{NO}$ exalado em indivíduos normotensos, mas não em pacientes com hipertensão sistêmica. ${ }^{(5)}$

Medidas diretas do NO exalado em pacientes com HP primária têm mostrado níveis similares com controles saudáveis, relacionando-se com a capacidade de difusão do pulmão. Isto sugere que a liberação basal de NO está ainda presente nessa condição patológica. Entretanto o desenvolvimento da HP secundária a doenças sistêmicas (esclerose sistêmica, insuficiência cardíaca crônica) parece associar-se com a diminuição da produção de $\mathrm{NO}$ exalado no repouso e durante o exercício. Em pacientes com insuficiência cardíaca e HP, o NO exalado é negativamente correlacionado com a resistência vascular pulmonar e com a baixa tensão de oxigênio venosa mista. Similarmente, embora a produção de NO seja geralmente aumentada nas vasculites sistêmicas, a redução é observada quando se desenvolve hipertensão pulmonar (HP). Estes achados aumentam a hipótese de que o $\mathrm{NO}$ expirado pode ser derivado em parte do endotélio. A redução do NO exalado na HP pode refletir o prejuízo da liberaçáo do $\mathrm{NO}$ endotelial associada com esta condição, ou o aumento da muscularização das artérias pulmonares distais (condiçâo tipicamente associada com a HP e que aumentaria o NO). ${ }^{(18)}$

Produtos de reaçóes bioquímicas do NO são correlacionados, inversamente, com as pressóes da artéria pulmonar em pacientes com HP primária e, com a duração da doença desde o seu diagnóstico. Isto pode refletir a redução da expressão da NOS3 em pacientes com HP. Baixos níveis de NO exalado em pacientes com HP poderia ser compatível com a redistribuição do fluxo sangüíneo dos capilares do septo alveolar para os vasos extra-alveolares. ${ }^{(5)}$

$\mathrm{O}$ NO exalado representa a produção de NO nos pulmóes e seus níveis podem estar aumentados na SARA, uma vez que essa produçáo está envolvida com mudanças nas atividades das formas constitutivas da NOS, representadas pelas isoformas nNOS e eNOS, afetadas pela acidose, alterando assim o $\mathrm{pH}$ intracelular. ${ }^{(24)}$

A ventilação mecânica pode contribuir para a lesão pulmonar e inflamaçáo. A liberaçáo de $\mathrm{NO}$ em pacientes que receberam ventilação mecânica pode entáo refletir a distensão alveolar, inflamação ou ambos. ${ }^{(25)}$ No estudo realizado por Gessner et al. o $\mathrm{NO}_{2}^{-}$do CEP está fortemente relacionado com o volume corrente (tidal volume $-\mathrm{VT}$ ), exibindo maior correlação com a extensão da lesão pulmonar. Em paciente com lesóes pulmonares graves altos níveis de $\mathrm{NO}_{2}^{-}$do CEP sáo encontrados, quando comparados com pacientes sem lesão pulmonar e ventilados com VT semelhantes. A forte relaçáo entre $\mathrm{NO}_{2}^{-}$do CEP e VT sugere que isto pode resultar de uma extensa distensão pulmonar devido à reduçáo funcional do volume pulmonar na SARA. Se $\mathrm{NO}_{2}$ - do CEP é relatado na distensáo pulmonar e se o volume pulmonar disponível está reduzido na lesão pulmonar grave, o aumento da relação $\mathrm{NO}_{2}$ - do $\mathrm{CEP} / \mathrm{VT}$ poderia refletir o aumento da distensão alveolar ${ }^{(25)}$ 
$\mathrm{O}$ interesse despertado pela inalação de $\mathrm{NO}$ em pacientes com SARA resulta da distribuição preferencial deste gás para as regiōes bem ventiladas e não colapsadas dos pulmóes. ${ }^{(26)} \mathrm{Em}$ virtude de seu efeito vasodilatador, o fluxo sanguíneo regional é redirecionado para essas áreas, com a conseqüente melhora da relação ventilação/perfusão $(\mathrm{V} / \mathrm{Q})$. Além disso, por ter meia-vida muito curta e ligar-se avidamente à hemoglobina, esses efeitos são muito fugazes, não ocasionando vasodilatação sistêmica. ${ }^{(26,27)}$ Estudos mostram significativa melhora na oxigenação e diminuição da pressão arterial pulmonar média em pacientes com SARA sob efeito de NO inalado. ${ }^{(28,29)}$ Essa forma de tratamento é diferente das até agora utilizadas, uma vez que os vasodilatadores usualmente promovem diminuição generalizada do tônus vasomotor, inclusive para as áreas não ventiladas, piorando a relação $\mathrm{V} / \mathrm{Q}$, além de ocasionarem hipotensão arterial sistêmica. ${ }^{(28)} \mathrm{O}$ uso continuado e controlado de $\mathrm{NO}$ a partir de fontes exógenas por períodos longos não promove taquifilaxia, nem efeitos deletérios maiores, podendo vir a tornar-se uma medida terapêutica importante para essa situação. ${ }^{(28,29)}$ Entretanto, a rápida melhoria obtida na relação $\mathrm{PaO}_{2} / \mathrm{FiO}_{2}{ }^{(29)}$ não é sustentada por muito tempo, nem promove mudanças significativas na mortalidade ou número de dias em ventilaçấo mecânica. ${ }^{(28,29)}$ Portanto, o real papel do NO inalado nesses quadros ainda precisa ser mais bem estabelecido. ${ }^{(28)}$

O estudo realizado por Adrie et al..$^{(9)}$ mostrou que pacientes com pneumonia, ventilados mecanicamente, têm níveis significativamente aumentados de $\mathrm{NO}$ exalado e nasal. Entretanto, não existe associação com o aumento da produção sistêmica de $\mathrm{NO}$, avaliados pelos níveis $\mathrm{NO}_{3}$ plasmático, quando comparados com pacientes com SARA ou outras formas agudas de falência respiratória. Embora os níveis de $\mathrm{NO}$ exalado em pacientes agudamente doentes, dependentes de assistência ventilatória mecânica, sejam muito baixos, os níveis de $\mathrm{NO}$ exalado em pacientes ventilados com pneumonia é relativamente elevado em relaçáo aos níveis vistos em pacientes sem pneumonia. A identificaçáo de valores limiares de $\mathrm{NO}$ exalado podem ajudar na distinção de pacientes com ou sem pneumonia. Este aumento foi associado com a alta produçáo nasal de $\mathrm{NO}$ em um mesmo grupo, sugerindo que este fenômeno reflete mais uma resposta epitelial generalizada, do que uma resposta específica das vias aéreas distais.

Corradi et al. ${ }^{(4)}$ observaram que pacientes com pneumonia comunitária apresentam aumentos de $\mathrm{NO}_{3}^{-}$no $\mathrm{CEP}$, na fase aguda da doença. Isso ocorre, pois nessa fase há aumento da produção de NO pelos pulmóes, devido a presença de macrófagos nos tecidos pulmonares que aumentam a expressáo das enzimas NOx sintase. Além disso, as bactérias presentes nas vias aéreas também podem afetar os níveis de $\mathrm{NO}_{3}^{-}$.

\section{Medida do condensado do exalado pulmonar e tera- pia intensiva}

A revisão especializada (MEDLINE) revela, claramente, que a terapia intensiva ainda não se convenceu da utilidade da colheita e análise do CEP. Quando se pesquisam os termos "exhaled breath condensate" e "critical care", "intensive care" e "mechanical ventilation" o resultado revela, respectivamente, 10,3 e 20 trabalhos indexados. Dentre esses trabalhos apenas poucos merecem destaque. Ao lado da medida dos metabólitos do $\mathrm{NO}$, a medida do $\mathrm{pH}$ e de $\mathrm{H}_{2} \mathrm{O}_{2}$ têm sido as mais destacadas.

Uma das possíveis utilidades do CEP em terapia intensiva é o de se testar possíveis procedimentos terapêuticos em pacientes com insuficiência respiratória aguda. Para exemplificar essa possibilidade relatamos a seguinte experiência. Em um pequeno número de pacientes, com lesão pulmonar aguda, a inalação de salbutamol diminuiu a acidose das vias aéreas, que é um marcador de inflamação, e associou-se a uma tendência em direção à diminuição de marcadores nitrosativos e marcadores de estresse oxidativo. Os benefícios da estimulação $\beta$-adrenérgica tem sido descrita em pacientes com lesōes pulmonares agudas. Biomarcadores no CEP têm sido usados para estudar os efeitos de salbutamol na inflamação pulmonar em pacientes mecanicamente ventilados com lesóes pulmonares aguda. A coleta do CEP foi realizada utilizando-se condensadores comercialmente disponíveis (por exemplo, o EcoScreen; Jaeger, Würzburg, Germany) ${ }^{\oplus}$, acoplado com adaptadores especiais (Por exemplo o VentAdapter; FILT Lung and Chest Diagnostic GmbH, Berlin, Germany) ${ }^{\oplus}$. Nessa investigação, o umidificador foi removido 1 minuto antes de se começar a coleção do CEP. O condensador resfriou o gás exalado a $-20^{\circ} \mathrm{C}$. A temperatura do coletor foi medida no início da coleta e o CEP (1 a $2 \mathrm{ml})$ foi coletado de 25 a 45 minutos, dependendo do volume por minuto de cada paciente. ${ }^{(30)}$ Embasado nesse trabalho dois pontos merecem destaque: a) Uma padronizaçáo de coleta aleatória e; b) O preço elevado dos condensadores comerciais (pesquisas utilizando a Internet).

A insuficiência respiratória aguda implica em reação inflamatória, e, descartando tecnologias mais sofisticadas e caras como, por exemplo, a dosagem de citocinas a medida do $\mathrm{pH}$ e do $\mathrm{H}_{2} \mathrm{O}_{2}$ têm se destacado para essa finalidade. A acidificaçáo do ar expirado e o aumento dos níveis de $\mathrm{H}_{2} \mathrm{O}_{2}$ são duas medidas que têm sido destacadas na literatura.

$\mathrm{O}$ pH do CEP é baixo em variadas doenças inflamatórias pulmonares, incluindo asma, DPOC, FC, pneumonia e SARA. Pelo fato das alteraçóes temporais do $\mathrm{pH}$ não serem ainda claras, Walsh et al. ${ }^{(31)}$ propuseram um método para a freqüente e intensiva medida do $\mathrm{pH}$ do CEP. Esses autores examinaram a coleta, padronização dos gases (remoção do 
$\mathrm{CO}_{2}$ ) e a monitorização contínua do pH do CEP na saída expiratória do respirador em pacientes sob ventilação mecânica. Esses autores desenvolveram um condensador que é conectado à saída expiratória e resfriado por um sistema elétrico. A medida do $\mathrm{pH}$ foi realizada a cada 6 segundos. Após testar a segurança do sistema, 19 pacientes pediátricos foram monitorizados por 6-96 horas. O pH do CEP tornou-se mais ácido durante os períodos de deterioração clínica e, normalizado nos períodos de recuperação. Essa experiência revela dois pontos importantes: a) A utilidade da medida do $\mathrm{pH}$ como monitorizaçáo da inflamação e, b) A dificuldade da coleta do CEP durante a assistência respiratória mecânica. O segundo fato foi plenamente constatado nos dois projetos por nós desenvolvidos no programa de pós-graduação do Departamento de Cirurgia e Anatomia da Faculdade de Medicina de Ribeirão Preto da Universidade Sáo Paulo (FMRP-USP), e tem sido uma das motivaçóes do prosseguimento das investigaçóes que nos levem à padronizaçáo e obtençáo de um sistema barato e eficiente para a coleta do CEP durante a ventilação mecânica.

A determinaçáo do conteúdo de $\mathrm{H}_{2} \mathrm{O}_{2}$ no CEP com a finalidade de explorar a sua relação com a intensidade da inflamação e prognóstico de pacientes sob ventilação mecânica, Yang et al. ${ }^{(32)}$ estudaram 36 pacientes coletando-se o CEP nos dias 1, 3, 5, 7 após a ventilação mecânica. $\mathrm{O} \mathrm{H}_{2} \mathrm{O}_{2}$ no CEP foi medido por fluorimetria observando-se uma queda nos níveis de $\mathrm{H}_{2} \mathrm{O}_{2}$ nos dias 3,5 e 7 de pacientes sobreviventes, diminuição essa em relação ao dia 1 após a ventilação mecânica. Observou-se uma diminuiçáo dos níveis de $\mathrm{H}_{2} \mathrm{O}_{2}$ no dia 7 quando comparado com o dia 3 após a ventilaçấo mecânica. Não houve diferença entre o dia 1 comparado com o dia 5. Nos pacientes que faleceram, observaram-se significantes diminuiçóes dos níveis de $\mathrm{H}_{2} \mathrm{O}_{2}$ nos dias 1,3 e 5 quando comparados com o dia 7 após ventilação mecânica. Não se observaram diferenças entre os dias 1 e 3 . Ocorreram diferenças significantes nos dia 1, 5 e 7 entre pacientes sobreviventes e não sobreviventes. Não se observaram correlaçóes entre os níveis de $\mathrm{H}_{2} \mathrm{O}_{2}$ e os dados do escore Acute Physiologic Chronic Health Evaluation (APACHE) II e APACHE III. Esses dados foram publicados por Yang $\&$ Wang e, embora a apresentação dos dados seja um pouco confusa, trata-se de uma experiência de serviço chinês, que levou a uma conclusão genérica. Os autores embasados em seus dados acham que a determinaçáo dos níveis de $\mathrm{H}_{2} \mathrm{O}_{2}$ no CEP correlaciona-se com a gravidade de pacientes submetidos à ventilação mecânica, podendo ser útil na monitorização da reação inflamatória das vias aéreas e, assim, orientar a terapêutica e o prognóstico após ventilação mecânica. ${ }^{(32)}$

Dentro do processo de revisão da literatura chamou a atenção uma investigação que teve a finalidade de estabelecer uma relação entre o VT e níveis de $\mathrm{NO}_{2}^{-}$medidos no CEP. É profundamente sabido que a própria ventilação mecânica pode lesar os pulmóes. Baixos VTs são desejáveis para prevenir lesóes pulmonares, mas são calculados com base nos pesos dos pacientes, o que pode levar à utilização de VT alto em relação a pulmóes "funcionalmente" pequenos na SARA. Assim, Gessner et al. ${ }^{(25)}$ hipotetizaram que a concentração do $\mathrm{NO}_{2}$ no CEP poderia aumentar em funçáo da distensão pulmonar. Realizaram um estudo prospectivo, não controlado, incluindo 35 pacientes com insuficiência respiratória aguda, pneumonias ou exacerbação de sintomas da DPOC. Além do $\mathrm{NO}_{2}^{-}$foram dosadas interleucinas e pró-calcitonina no CEP. Observaram-se correlaçóes entre esses parâmetros da inflamação com os níveis de $\mathrm{NO}_{2}^{-}$do CEP. Além disso, a relação $\mathrm{NO}_{2}^{-} / \mathrm{VT}$ foi correlacionada diretamente com a lesão pulmonar, concluindo-se que o $\mathrm{NO}_{2}{ }^{-}$aumentou linearmente com o VT adotado de acordo com os pés dos pacientes. $\mathrm{O}$ aumento dessa relação revela, portanto, um aumento inapropriado do $\mathrm{NO}_{2}^{-}$significando um estresse adicional das unidades respiratórias ainda funcionais. A relação $\mathrm{NO}_{2}^{-/ V T}$ poderia se um parâmetro para identificação de situações de estresse mecânico induzido pelo respirador. Os resultados desse estudo sugerem uma correlação muito boa entre $\mathrm{o} \mathrm{NO}_{2}^{-}$do CEP e a distensão pulmonar, sem nenhuma correlação dos parâmetros de inflamação sistêmica ou pulmonar. A distensáo inapropriada pode ser a conseqüência de aumento do desrecrutamento alveolar com aumento da lesão pulmonar. A real utilidade da relaçáo $\mathrm{NO}_{2}{ }^{-} / \mathrm{VT}$ merece estudos adicionais como parâmetro da monitorização de pacientes submetidos à ventilação mecânica.

\section{COMENTÁRIOS}

A técnica do CEP destaca-se pela possibilidade de ser segura, simples e de baixo custo, não necessitando de treino específico exaustivo. Porém, ainda não está bem padronizada, apresentando algumas e variadas limitaçóes. Certamente, nas técnicas para medida "offline" do NO (nitrito/nitrato no CEP), o fator limitante mais importante é o veículo usado para a condensaçáo do ar exalado. A grande maioria dos trabalhos utiliza o nitrogênio líquido, um gás altamente explosivo, com custo elevado e que requer cuidados especiais no seu transporte e armazenamento, inviabilizando seu uso em muitos ambientes hospitalares. Além disso, a diluição dos biomarcadores nas amostras, a contaminação das amostras pela saliva e a temperatura de condensação também atuam como fatores limitantes. ${ }^{(28)}$

Assim, devido à necessidade dispor de uma técnica com melhor custo benefício foram desenvolvidas duas dissertaçóes de mestrado no programa de pós-graduação do Departamen- 
to de Cirurgia e Anatomia da FMRP-USP. Nesses projetos foram testados dois dispositivos montados artesanalmente em caixas de isopor. Cada dispositivo consistiu de uma extensão plástica atóxica e transparente com 100 centímetros $(\mathrm{cm})$ de comprimento e $0,1 \mathrm{~cm}$ de espessura, com tubo Eppendorf afixado na sua porção final para coleta do condensado exalado. Um bocal foi adaptado ao circuito, para que o indivíduo respirasse seu VT através dele. Para a condensação do ar exalado foi utilizado gelo picado com sal grosso (proporção de 6 partes de gelo para 3 partes de sal grosso), ou gelo seco com gelo picado, sendo que o gelo seco foi acondicionado em uma caixa plástica, ficando separado do gelo picado. A temperatura nos dois sistemas ficou, respectivamente, em torno de -10 e $-15^{\circ}$ C. Após a coleta, as amostras foram armazenadas a $-70^{\circ} \mathrm{C}$. Participaram deste estudo 28 voluntários adultos, de ambos os sexos, com idade entre 26 e 71 anos, os quais foram distribuídos em dois grupos denominados grupo controle não-cirúrgico $(\mathrm{CNC})$ e grupo controle cirúrgico $(\mathrm{CC})$.

Os volumes de CEP coletados nos dois métodos, não apresentaram diferenças significativas nas comparaçóes realizadas intragrupos e intergrupos. No entanto, foi possível constatar que o método de gelo e sal grosso proporcionou a coleta de volumes maiores de condensado quando comparado ao método do gelo seco. Esse dado foi muito interessante, pois liberou a metodologia da utilização, nem sempre disponível, do gelo seco.

Observou-se que no pós-operatório tardio (24 horas) de cirurgia valvar, e na cirurgia de revascularização do miocárdio os níveis de $\mathrm{NO}_{2}^{-}$exalado diminuíram ou permaneceram próximos aos valores do pré-operatório. Verificaram-se, ainda, valores médios aumentados no pós-operatório tardio de pacientes submetidos à revascularização do miocárdio, quando as amostras foram resfriadas com gelo seco. Segundo Goldoni et al. ${ }^{(33)}$ baixas temperaturas levam à condensação de uma grande quantidade de moléculas de água, com conseqüente diluiçáo das concentraçóes de $\mathrm{NO}_{2}$. As menores temperaturas foram encontradas no resfriamento com gelo e sal grosso, o que pode justificar as menores concentraçóes de $\mathrm{NO}_{2}{ }^{-}$presentes nas amostras coletadas por este método quando comparadas com as coletas com gelo e gelo seco, onde foram observadas as maiores concentrações.

Esses achados foram muito interessantes, mostrando que é possível diminuir o custo benefício da técnica do condensado do exalado. Já em relação ao período em que os pacientes se encontravam em ventilação mecânica, o condensado exalado não foi coletado para todos os indivíduos, devido a problemas com a temperatura de congelamento ou até mesmo com a própria metodologia. Por esse motivo mais ensaios serão necessários nessa área para que se descubra o melhor método de coleta durante a assistência ventilatória.

\section{CONCLUSÃO}

Estudos específicos e tecnologias ainda são necessários para que o CEP se torne uma ferramenta rotineira de pesquisas e monitorização de pacientes gravemente enfermos com comprometimento do sistema respiratório. A literatura aponta para a realização de esforços nessa direção. Já existe a previsão de que em um futuro próximo se incorpore a medida do $\mathrm{NO}$ e pH como parâmetros da assistência respiratória como as já consagradas oximetria e capnometria ${ }^{(34)}$. Nesse caso dois aspectos técnicos precisam de decisóes, levando-se em conta que a medida do NO exalado já é possível com eletrodos especiais e, que a utilizaçáo do CEP permite o armazenamento de amostras para posterior dosagem. Enquanto isso, o nosso maior desafio pessoal é a padronização da coleta do CEP durante a ventilação mecânica, padronização essa que implica na obtençáo de um sistema seguro e de baixo custo.

Como sugestão final dessa revisão recomenda-se a consulta do resultado sobre as orientaçóes relacionadas ao CEP, publicadas em 2005, como resultado de um comitê conjunto da American Thoracic Association (ATS) e da European Respiratory Society (ERS). ${ }^{(35)}$

Agradecimentos: Trabalho realizado com apoio da Fundação de Amparo a Pesquisa do Estado de São Paulo (FAPESP), Brasil e da FAEPA - Fundação de Apoio ao Ensino, Pesquisa e Assistência do Hospital das Clínicas da Faculdade de Medicina de Ribeirão Preto da Universidade de São Paulo - USP - Ribeirão Preto (SP), Brasil.

\section{ABSTRACT}

Endogenous production of nitric oxide can be detected and monitored in exhaled air of men and animals. The main objective of this review was to discuss if nitric oxide in exhaled breath condensate is a useful tool when investigating respiratory dysfunctions in intensive care units. Specialized literature reveals an increasing interest in the use of exhaled breath condensate as a non-invasive method to investigate pulmonary disease. However, a standardized method for its collection is lacking, and use of different methods of respiratory support complicates comparison among different studies. In addition, the same specialized literature review emphasized possible difficulties for routine use of the exhaled breath condensate in intensive care patients, mainly under mechanical ventilation. Until exhaled breath condensate becomes a routine tool of research and monitoring of intensive care patients, more specific studies and technologies are still necessary. Its importance has been related to physiological control of the pulmonary function and to physiopathology of pulmonary disease involving chronic inflammation and oxidative stress.

Keywords: Nitric oxide/diagnostic use; Respiratory tract diseases/diagnosis; Intensive care units 


\section{REFERÊNCIAS}

1. Ignarro LJ, Buga GM, Wood KS, Byrns RE, Chaudhuri G. Endothelium-derived relaxing factor produced and released from artery and vein is nitric oxide. Proc Natl Acad Sci U S A. 1987;84(24):9265-9.

2. Palmer RM, Ferrige AG, Moncada S. Nitric oxide release accounts for the biological activity of endothelium-derived relaxing factor. Nature. 1987;327(6122):524-6.

3. Ricciardolo FL, Sterk PJ, Gaston B, Folkerts G. Nitric oxide in health and disease of the respiratory system. Physiol Rev. 2004;84(3):731-65. Review.

4. Corradi M, Pesci A, Casana R, Alinovi R, Goldoni M, Vettori MV, Cuomo A. Nitrate in exhaled breath condensate of patients with different airway diseases. Nitric Oxide. 2003;8(1):26-30.

5. Kharitonov S, Barnes PJ. Exhaled markers of pulmonary disease. Am J Respir Crit Care Med. 2001;163(7):1693722.

6. Biban P, Zangardi T, Baraldi E, Dussini N, Chiandetti L, Zacchello F. Mixed exhaled nitric oxide and plasma nitrites and nitrates in newborn infants. Life Sci. 2001;68(25):2789-97.

7. Ellis G, Adatia I, Yazdanpanah M, Makela SK. Nitrite and nitrate analyses: a clinical biochemistry perspective. Clin Biochem. 1998;31(4):195-220.

8. Ojoo JC, Mulrennan SA, Kastelik JA, Morice AH, Redington AE. Exhaled breath condensate $\mathrm{pH}$ and exhaled nitric oxide in allergic asthma and in cystic fibrosis. Thorax. 2005;60(1):22-6. Comment in: Thorax. 2005;60(1):2-3. Thorax. 2005;60(7):614; author reply 614.

9. Adrie C, Monchi M, Dinh-Xuan AT, Dall'Ava-Santucci J, Dhainaut FJ, Pinsky MR. Exhaled and nasal nitric oxide as a marker of pneumonia in ventilated patients. Am J Respir Crit Care Med. 2001;163(5):1143-9.

10. Förstermann U, Trogisch G, Busse R. Species-dependent differences in the nature of endothelium-derived relaxing factor. Eur J Pharmacol. 1984;106(3):639-43.

11. Chatkin JM, Djupesland P, Qian W, Haight J, Zamel N. Óxido nítrico exalado no diagnóstico e acompanhamento das doenças respiratórias. J Pneumol. 2000;26(1):36-43.

12. Cerqueira NF, Yoshida WB. Óxido nítrico: revisão. Acta Cir Bras. 2002;17(6):417-23.

13. Kelm M. Nitric oxide metabolism and breakdown. Biochim Biophys Acta. 1999;1411(2-3):273-89.

14. Mutlu GM, Garey KW, Robbins RA, Danziger LH, Rubinstein I. Collection and analysis of exhaled breath condensate in humans. Am J Respir Crit Care Med. 2001;164(5):731-7.

15. Kharitonov SA, Barnes PJ. Exhaled biomarkers. Chest. 2006;130(5):1541-6.

16. Effros RM, Dunning MB 3rd, Biller J, Shaker R. The promise and perils of exhaled breath condensates. Am J
Physiol Lung Cell Mol Physiol. 2004;287(6):L1073-80. Review.

17. De Lema JB, González M, Vigil L, Casan P. [Exhaled breath condensate: standardized collection of samples from healthy volunteers]. Arch Bronconeumol. 2005;41(10):5846. Comment in: Arch Bronconeumol. 2005;41(10):540-1. Spanish.

18. Clini E, Ambrosino N; INOC. Italian Nitric Oxide Club. Nitric oxide and pulmonary circulation. Med Sci Monit. 2002;8(8):RA178-82. Review.

19. Kharitonov SA. Exhaled markers of inflammatory lung diseases: ready for routine monitoring? Swiss Med Wkly. 2004;134(13-14):175-92. Comment in: Swiss Med Wkly. 2004;134(31-32):468; author reply 468.

20. Recommendations for standardized procedures for the on-line and off-line measurement of exhaled lower respiratory nitric oxide and nasal nitric oxide in adults and children-1999. This official statement of the American Thoracic Society was adopted by the ATS Board of Directors, July 1999. Am J Respir Crit Care Med. 1999;160(6):2104-17. Comment in: Am J Respir Crit Care Med. 2002;165(8):1188; author reply 1188.

21. Kharitonov S, Alving K, Barnes PJ. Exhaled and nasal nitric oxide measurements: recommendations. The European Respiratory Society Task Force. Eur Respir J. 1997;10(7):1683-93.

22. Hedenstierna G, Högman M. Can exhaled NO be used as a marker of airway inflammation? Eur Respir J. 1998;12(6):1248-9.

23. Brett SJ, Evans TW. Measurement of endogenous nitric oxide in the lungs of patients with the acute respiratory distress syndrome. Am J Respir Crit Care Med. 1998;157(3 Pt 1):993-7.

24. Pedoto A, Caruso JE, Nandi J, Oler A, Hoffmann SP, Tassiopoulos AK, et al. Acidosis stimulates nitric oxide production and lung damage in rats. Am J Respir Crit Care Med. 1999;159(2):397-402.

25. Gessner C, Hammerschmidt S, Kuhn H, Lange T, Engelmann L, Schauer J, Wirtz H. Exhaled breath condensaten and its relation to tidal volume in acute lung injury. Chest. 2003;124(3):1046-52.

26. Payen DM. Is nitric oxide inhalation a "cosmetic" therapy in acute respiratory distress syndrome? Am J Respir Crit Care Med. 1998;157(5 Pt 1):1361-2. Comment on: Am J Respir Crit Care Med. 1998;157(5 Pt 1):1372-80.

27. Saleh D, Barnes PJ, Giaid A. Increased production of the potent oxidant peroxynitrite in the lungs of patients with idiopathic pulmonary fibrosis. Am J Respir Crit Care Med. 1997;155(5):1763-9.

28. Dellinger RP, Zimmerman JL, Taylor RW, Straube RC, Hauser DL, Criner GJ, et al. Effects of inhaled nitric oxide in patients with acute respiratory distress syndrome: results of a randomized phase II trial. Crit Care 
Med. 1998;26(1):15-23. Comment in: Crit Care Med. 1998;26(1):1-2. Crit Care Med. 1998;26(1):2-3. Crit Care Med. 1999;27(4):846-7. Crit Care Med. 1999;27(4):8478. Crit Care Med. 1999;27(4):848-9.

29. Rossaint R, Falke KJ, López F, Slama K, Pison U, Zapol WM. Inhaled nitric oxide for the adult respiratory distress syndrome. N Engl J Med. 1993;328(6):399-405. Comment in: N Engl J Med. 1993;328(6):431-2. N Engl J Med. 1993;329(3):206-7. N Engl J Med. 1993;329(3):206; author reply 207.

30. Roca O, Gómez-Ollés S, Cruz MJ, Muñoz X, Griffiths MJ, Masclans JR. Effects of salbutamol on exhaled breath condensate biomarkers in acute lung injury: prospective analysis. Crit Care. 2008;12(3):R72.

31. Walsh BK, Mackey DJ, Pajewski T, Yu Y, Gaston BM, Hunt JF. Exhaled-breath condensate $\mathrm{pH}$ can be safely and continuously monitored in mechanically ventilated patients. Respir Care. 2006;51(10):1125-31. Comment in: Respir Care. 2006;51(10):1118-9.

32. Yang GH, Wang GF. [Determination of the hydrogen peroxide level in exhaled breath condensate of patients under mechanical ventilation]. Zhongguo Wei Zhong Bing Ji Jiu Yi Xue. 2008;20(6):324-6. Chinese.

33. Goldoni M, Caglieri A, Andreoli R, Poli D, Manini P, Vettori MV, et al. Influence of condensation temperature on selected exhaled breath parameters. BMC Pulm Med. 2005;5:10.

34. Owens RL, Stigler WS, Hess DR. Do newer monitors of exhaled gases, mechanics, and esophageal pressure add value? Clin Chest Med. 2008;29(2):297-312, vi-vii.

35. Horváth I, Hunt J, Barnes PJ, Alving K, Antczak A, Baraldi E, Becher G, van Beurden WJ, Corradi M, Dekhuijzen R, Dweik RA, Dwyer T, Effros R, Erzurum S, Gaston B, Gessner C, Greening A, Ho LP, Hohlfeld J, Jöbsis Q, Laskowski D, Loukides S, Marlin D, Montuschi P, Olin AC, Redington AE, Reinhold P, van Rensen EL, Rubinstein I, Silkoff P, Toren K, Vass G, Vogelberg C, Wirtz H; ATS/ ERS Task Force on Exhaled Breath Condensate. Exhaled breath condensate: methodological recommendations and unresolved questions. Eur Respir J. 2005;26(3):523-48. Comment in: Eur Respir J. 2005;26(3):371-2. Eur Respir J. 2006;28(1):251-2; author reply 252-3. 